\title{
ON THE SCALAR POTENTIAL MODELS FROM THE ISOSPECTRAL POTENTIAL CLASS
}

\author{
V. Gomes Lima ${ }^{(a)}$, V. Silva $\operatorname{Santos}^{(b)}$ and R. de Lima Rodrigues ${ }^{(c) *}$ \\ (a,c) Centro Brasileiro de Pesquisas Físicas (CBPF) \\ Rua Dr. Xavier Sigaud, 150 \\ CEP 22290-180, Rio de Janeiro-RJ, Brazil \\ (a) Departamento de Física, Universidade Federal Rural do Rio de Janeiro \\ Antiga Rodovia Rio-São Paulo Km 47, BR 465 \\ CEP 23.890-000, Seropédica-RJ - Brazil \\ (b) Departamento de Engenharia Civil, Universidade Federal de Campina Grande \\ CEP 58.109-970, Campina Grande - PB - Brazil
}

\begin{abstract}
Static field classical configurations in $(1+1)$-dimensions for new non-linear potential models are investigated from an isospectral potential class and the concept of bosonic zero- mode solution. One of the models here considered has a static non-topological configuration with a single vacuum state that breaks supersymmetry.
\end{abstract}

PACS numbers: 11.30.Pb, 03.65.Fd, 11.10.Ef

*Permanent address: Departamento de Ciências Exatas e da Natureza, Universidade Federal de Campina Grande, Cajazeiras - PB, 58.900-000 - Brazil, e-mail: rafaelr@cbpf.br ou rafael@cfp.ufpb.br 


\section{INTRODUCTION}

Rencently, static classical configurations that are exact solution of the equation of motion in scalar potential model have drawn much attention mainly because they have found interesting applications as a solitary wave [1]. There are several methods to build up soliton (kink) solutions associated with some nonlinear differential equations [2]. The kink of a scalar field theory is a static, non-singular, classically stable and finite-localized energy solution of the equation of motion [3]. When $\phi(x, t)=f(x-v t)$, a kink solution is called solitary wave. The role of classical solutions in quantum field theories have recently been overviewed [4]. There, one can see how a quantum field theory has topological and non-topological soliton solutions in higher spatial dimensions. In the literature, there is a surprising number of scalar potential models in higher spatial dimensions with exactly solvable equations of motion. Also, a variety of classical finite-energy static solutions are known.

The connection between supersymmetry in quantum mechanics (SUSY QM) [5 90 and the topological and non-topological solitons in terms of a scalar potential (for the case of a single field) has been discussed in the literature [11 15]. The connection between SUSY QM associated with two-component eigenfunctions [9] and the topological solitons in terms of two coupled scalar fields have already been considered in [10]. Recently, the reconstruction of 2-dimensional scalar field potential models has been considered starting from the Morse and the Scarf II hyperbolic potential, and quantum corrections to the solitonic sectors of both potentials are pointed out [16].

In the present work, from a non-polynomial potential in supersymmetric quantum mechanics, we shall propose new potentials as functions of one single real scalar field. Indeed, in $(1+1)$ dimensions, non-polynomial interactions are not really harmful, from the point of view of renormalisation. Actually, non-linear $\sigma$-models are the most representative class of renormalisable models in 2D, and their interactions are described by non-polynomial functions. We consider the interesting program of proposing new potential models in $1+1$ dimensions, whose essential point is associated with the translational invariance of the static field configuration [11]. Here, the aim is to find field potential models via SUSY QM considering a one-dimensional quantum mechanical isospectral potential class such that the corresponding fluctuation operator sets a Schrödinger-like eigenvalue problem as a stability equation exactly solvable for the $\phi^{4}$-model. However, the corresponding field potential cannot be put in a closed form. Indeed, we show that in such a procedure there appears a new non-polynomial field potential model with infinite-energy static configuration, so that such a new potential may not be considered as a well-defined theoretical field potential model.

Actually, we shall bring two constructions of classical solutions. In the first case, we obtain the well-known topological kink, whereas in the second case we obtain a non-topological static configuration which is not a kink. In the second case the new static field configuration is not a smooth function over all the the spatial-time.

We also propose some pictures of the field potential models (figures I and III) and the static classical field configurations (figures II and IV). 


\section{STATIC CLASSICAL CONFIGURATION}

Consider the Lagrangian density for a single scalar field, $\phi(x, t)$, in $(1+1)$-dimensions, in natural system $(c=1=\hbar)$, given by

$$
\mathcal{L}\left(\phi, \partial_{\mu} \phi\right)=\frac{1}{2} \partial_{\mu} \phi \partial^{\mu} \phi-V(\phi)
$$

where $V(\phi)$ is any positive semi-definite function of $\phi$, which must have at least two zeros for kinks to exist. It represents a well-behaved potential energy. However, as it will be shown below, we have found a new potential which is exactly solvable exactly in the context of the classical theory in $(1+1)-\mathrm{D}$ that not a kink.

The field equation for a static classical configuration, $\phi=\phi_{c}(x)$, becomes

$$
-\frac{d^{2}}{d x^{2}} \phi_{c}(x)+\frac{d}{d \phi_{c}} V\left(\phi_{c}\right)=0, \quad \dot{\phi}_{c}=0
$$

with the following boundary conditions: $\phi_{c}(x) \rightarrow \phi_{\text {vacuum }}(x)$ as $x \rightarrow \pm \infty$. Since the potential is positive, it can be written as

$$
V(\phi)=\frac{1}{2} U^{2}(\phi)
$$

giving the well-known Bogomol'nyi condition,

$$
\frac{d \phi}{d x}= \pm U(\phi)
$$

where the solutions with the plus and minus signs represent two configurations.

\section{STABILITY EQUATION AND NEW POTENTIALS}

The classical stability of the static solution is investigated by considering small perturbations around it,

$$
\phi(x, t)=\phi_{c}(x)+\eta(x, t)
$$

where we expand the fluctuations in terms of the normal modes,

$$
\eta(x, t)=\sum_{n} \epsilon_{n} \eta_{n}(x) e^{i \omega_{n} t}
$$

the $\epsilon_{n}^{\prime} \mathrm{s}$ are so chosen that $\eta_{n}(x)$ are real. A localised classical configuration is said to be dynamically stable if the fluctuation does not destroy it. The equation of motion becomes a Schrödinger-like equation, viz.,

$$
F \eta_{n}(x)=\omega_{n}^{2} \eta_{n}(x), \quad F=-\frac{d^{2}}{d x^{2}}+V^{\prime \prime}\left(\phi_{c}\right)
$$

According to (3), one obtains the supersymmetric form, 15 


$$
V^{\prime \prime}\left(\phi_{c}\right)=U^{\prime 2}\left(\phi_{c}\right)+U\left(\phi_{c}\right) U^{\prime \prime}\left(\phi_{c}\right),
$$

where the primes stand for a second derivative with respect to the argument.

If the normal modes of (7) satisfy $\omega_{n}^{2} \geq 0$ the stability of the Schrödinger-like equation is ensured. Now, we are able to implement a method that provides a new potential from the potential term that appears in the fluctuation operator.

Next, we consider the following generalized isospectral potential as being the potential term (8) for the fluctuation operator:

$$
\begin{aligned}
V(x ; \alpha, \beta) & =m^{2}\left[3 \tanh ^{2}\left(\frac{m}{\sqrt{2}} x\right)-1\right] \\
& +2 m^{2} \beta\left[\operatorname{sech}^{4}\left(\frac{m}{\sqrt{2}} x\right)\left(2 \tanh \left(\frac{m}{\sqrt{2}} x\right)+\frac{\beta}{2} \operatorname{sech}^{4}\left(\frac{m}{\sqrt{2}} x\right) \chi\right) \chi\right], \\
\chi & =\chi(x ; \alpha, \beta)=\left\{\alpha+\beta\left[\tanh \left(\frac{m}{\sqrt{2}} x\right)-\frac{1}{3} \tanh ^{3}\left(\frac{m}{\sqrt{2}} x\right)\right]\right\}^{-1},
\end{aligned}
$$

where $\alpha$ and $\beta$ are constant parameters. This non-polynomial potential satisfies the condition $\omega_{n}^{2} \geq 0$, and the ground state associated to the zero mode $\left(\omega_{0}^{2}=0\right)$ is given by

$$
\eta^{(0)}(x ; \alpha, \beta)=N \chi(x) \operatorname{sech}^{2}\left(\frac{m}{\sqrt{2}} x\right),
$$

where $N$ is the normalization constant. Note that, if $|\alpha|>\frac{2 \beta}{3}$, the eigenfunction of the ground state is non-singular. This ground state was independently found by two authors [11,12] for $\beta=\frac{1}{2} \sqrt{\frac{3 m}{\sqrt{2}}}$.

It is well-known that the bosonic zero-mode eigenfunction of the stability equation is related with the kink by

$$
\eta^{(0)}(x ; \alpha, \beta) \propto \frac{d}{d x} \phi_{c}(x),
$$

so that, a priori, we may find the static classical configuration by a first integration. Therefore, the potential

$$
V(\phi ; \alpha, \beta)=\frac{1}{2}\left(\frac{d \phi}{d x}\right)^{2}
$$

yields a class of scalar potentials, $V(\phi)=V(\phi ; \alpha, \beta)$, which have exact solutions.

There, we can find various static field configurations; however, let us consider here only two cases.

Case (i): $\beta=0$ and $\alpha=2 N \sqrt{\frac{\sqrt{2}}{3 m}}$

In this case, $\chi(x) \rightarrow \frac{1}{\alpha}$, so that from (9) and (10), we obtain the following bosonic zero-mode solution:

$$
\eta^{(0)}(x)=\frac{1}{2} \sqrt{\frac{3 m}{\sqrt{2}}} \operatorname{sech}^{2}\left(\frac{m}{\sqrt{2}} x\right),
$$

and the non-polynomial potential becomes 


$$
V_{1-}(x)=m^{2}\left[3 \tanh ^{2}\left(\frac{m}{\sqrt{2}} x\right)-1\right] .
$$

Notice that the two Schrödinger-like fluctuation operators associated with both $V(x ; \alpha, \beta)$ and $V(x)$ non-polynomial potentials are positive semi-definite and completly isospectrals. However, their factorization has been implemented from distinct superpotentials. Indeed, while the Ricatti equation,

$$
V_{1-}(x)=W_{1}^{2}(x)+W_{1}^{\prime}(x)
$$

where $W^{\prime}(x)=\frac{d}{d x} W(x)$, has a particular solution given by

$$
W_{1}(x)=-\sqrt{2} m \operatorname{tgh}\left(\frac{m}{\sqrt{2}} x\right)
$$

provides

$$
\eta_{1-}^{(0)}(x)=e^{\int W_{1}(y) d y}=\frac{1}{2} \sqrt{\frac{3 m}{\sqrt{2}}} \operatorname{sech}^{2}\left(\frac{m}{\sqrt{2}} x\right)=\eta^{(0)}(x),
$$

with $\eta^{(0)}(x)$ given by Eq. (13), the Ricatti equation

$$
V_{0-}=V(x ; \alpha, \beta)=W_{0}^{2}(x)+W_{0}^{\prime}(x)
$$

has a particular solution given by $W_{0}(x)=\frac{d}{d x} \ell n\left(\eta_{0}(x ; \alpha, \beta)\right)$.

By substituting Eq. (13) in Eq. (11), we get the well-known kink of the double-well potential,

$$
\phi_{k}(x)=\frac{m}{\sqrt{\lambda}} \tanh \left(\frac{m}{\sqrt{2}} x\right) .
$$

When we express the position coordinate in terms of the kink, i.e. $x=x\left(\phi_{k}\right)$, we find the $\phi^{4}$-potential model with spontaneously broken symmetry in scalar field theory, viz.,

$$
V(\phi)=\frac{\lambda}{4}\left(\phi^{2}-\frac{m^{2}}{\lambda}\right)^{2}
$$

The mass of the kink is finite, but, in the next case, we obtain an undefined kink mass. Such a classical configuration cannot represents a stable particle. The pictures of the potential (19) and of the kink (18) are in Figures I and II. Note that both are smooth functions of the field and the spatial coordinate, respectively.

Case (ii): $\alpha=0$.

In this case, from (9), (10) and (11), we obtain the following non-polynomial potential in the singularity region associated to the ground state given by Eq. (10):

$$
\tilde{V}(\phi)=\frac{\lambda}{2}\left(1+3 e^{-\gamma \phi}\right)\left(1-\frac{2}{3} e^{\gamma \phi}\right)^{2}
$$

where $\gamma=\frac{2}{\sqrt{3}}$ and $\lambda$ is a dimensionless constant. 
The vacuum state, $\phi_{v}$, is given by

$$
\phi_{v}=\frac{\sqrt{3}}{2} \ln \left(\frac{3}{2}\right)
$$

This non-polynomial potential does not have the discrete symmetry, $\phi \rightarrow-\phi$, and there exists only one vacuum state, so that it is non-topological.

From (3), (4) and (20), for the minus sign of the Bogomol'nyi condition and the coupling constant $\lambda \neq 0$, the static classical configuration has the following explicit form

$$
\phi_{c}(x)=\frac{\sqrt{3}}{2} \ln \left(\frac{\tanh ^{2}(\sqrt{\lambda} x)}{1-\frac{1}{3} \tanh ^{2}(\sqrt{\lambda} x)}\right)
$$

where the integration constant is taken to be zero. Note that this static configuration satisfies Eq. (11) and the following boundary conditions $\phi_{c}(x) \rightarrow \phi_{v}$ as $x \rightarrow \pm \infty$. The pictures of the non-polynomial potential and the static solution are in Figs. II and IV. However, in Fig. IV, we have plotted this static field configuration only for the region in that it has a singularity in the origin.

The energy density for the static solution for the non-polynomial potential is given by

$$
E(x) \propto\left\{\sinh ^{2}(\sqrt{2} m x)\left(1-\frac{1}{3} \tanh ^{2}\left(\frac{m}{\sqrt{2}} x\right)\right)^{2}\right\}^{-1}
$$

which yields an undefined total energy or classical mass. Here, we have used the explicit relations between the static configuration and one-dimensional spatial coordinate.

\section{BROKEN SUSY QM}

In this section, we consider the fluctuation operator in the context of supersymmetry in Quantum Mechanics (SUSY QM), where the supersymmetric partners are build up from the stability equation. In $N=2$-SUSY, we define the following first order differential operators:

$$
A_{2}^{ \pm}= \pm \frac{d}{d x}+W_{2}(x), \quad A_{2}^{+}=\left(A_{2}^{-}\right)^{\dagger}
$$

The fluctuation operator for the bosonic sector is given by

$$
F_{2-} \equiv A_{2}^{+} A_{2}^{-}=-\frac{d^{2}}{d x^{2}}+V_{2-}(x), \quad V_{2-}(x)=\tilde{V}_{\mid \phi=\phi_{c}}^{\prime \prime},
$$

so that in terms of the superpotential we obtain the following nonlinear first order differential equation

$$
V_{2-}(x)=W_{2}^{2}(x)+W_{2}^{\prime}(x) \equiv V(x ; 0, \beta),
$$

where the prime means a derivative with respect to $x$. 
The superpotential that solve this Riccati equation for the non-polynomial potential has the following explicit form:

$$
W_{2}(x)=-\frac{m}{\sqrt{2}} \frac{\tanh ^{4}\left(\frac{m}{\sqrt{2}} x\right)+3}{\tanh \left(\frac{m}{\sqrt{2}} x\right)\left[3-\tanh ^{2}\left(\frac{m}{\sqrt{2}} x\right)\right]}
$$

Note that this particular solution to the Riccati differential equation has the following asymptotic behavior: $W_{2}(x) \rightarrow-\sqrt{2} m$ as $x \rightarrow \infty$ and $W_{2}(x) \rightarrow \sqrt{2} m$ as $x \rightarrow-\infty$. The supersymmetric partner of $F_{2-}$ is given by

$$
\begin{gathered}
F_{2+}=A_{2}^{-} A_{2}^{+}=-\frac{d^{2}}{d x^{2}}+V_{2+}(x) \\
V_{2+}(x)=W_{2}^{2}(x)-W_{2}^{\prime}(x)=m^{2}\left\{1+\tanh ^{2}\left(\frac{m}{\sqrt{2}} x\right)\right\} .
\end{gathered}
$$

These fluctuation operators are isospectral and consist of a pair Schrödinger-like Hamiltonians of Witten's model of broken SUSY [5]. Note that the shape invariance condition [6, 8] is not satisfied for $V_{ \pm}$given by Eqs. (26) and (28), i.e. $V_{2+}\left(x ; a_{2}\right) \neq V_{2-}\left(x ; a_{1}\right)+R$, where $a_{1}, a_{2}$ and $R$ are constants.

The eigenvalue equations for the supersymmetric partners $F_{\mp}$ are given by

$$
F_{2 \pm} \eta_{2 \pm}^{(n)}(x)=\omega_{2 \pm}^{(n)} \eta_{2 \pm}^{(n)}(x), \quad \omega_{2-}^{(n)}=\omega_{n}^{2}, \quad \omega_{0}^{2}=0
$$

for which, in general, $F_{2-}$ may have as eigenstates the well-known normal modes. However, when $\alpha=0$, the bosonic zero-mode $\left(\omega_{0}^{2}=0\right)$ satisfies the annihilation condition

$$
A_{2}^{-} \eta_{2-}^{(0)}=0 \Rightarrow \eta_{2-}^{(0)}(x) \propto \frac{1}{\sinh (\sqrt{2} m x)\left(2+\operatorname{sech}^{2}\left(\frac{m}{\sqrt{2}} x\right)\right)}=\eta^{(0)}(x ; 0, \beta) .
$$

This eigensolution is not normalizable, so that the fluctuation operator for the bosonic sector does not have a zero-mode. In this case, the integral $\int_{-\infty}^{+\infty}\left(\eta_{2-}^{(0)}(x)\right)^{2} d x$ is undefined. This result is in agreement with Eq. (10), for $\alpha=0$. Furthermore, bosonic zero mode satisfies $\eta_{2-}^{(0)}(x)=\frac{d}{d x} \phi_{c}(x)$. The fermionic sector fluctuation operator $F_{2+}$ does not have zero-modes because $\eta_{2+}^{(0)}$,

$$
A_{2}^{+} \eta_{2+}^{(0)}=0 \Rightarrow \eta_{2+}^{(0)} \propto \sinh (\sqrt{2} m x)\left[2+\operatorname{sech}^{2}\left(\frac{m}{\sqrt{2}} x\right)\right]
$$

because it is not normalizable. In this case we have broken SUSY. Indeed, it is easy to see that

$$
\int_{-\infty}^{+\infty}\left(\eta_{2+}^{(0)}(x)\right)^{2} d x \rightarrow \infty
$$

The eigenvalues $\omega_{ \pm}$and eigenfunctions $\eta_{2 \pm}^{(n)}$ can be exactly solved in a similar way for a general potential. All eigenvalues of $F_{2+}$ are eigenvalues of $F_{2-}$, i.e. $\omega_{2-}^{(n)}=\omega_{2+}^{(n)}>0$, so that the ground state and the excited state of both $F_{2 \pm}$ have energy different from zero. 


\section{CONCLUSION}

In this work, we investigate the classical stability of a new isospectral non-polynomial potential model with static classical configuration which solve exactly the equation of motion.

Indeed, the classical finite-energy static solutions appear in field theoretical models with spontaneously broken symmetry (SUSY), for example, in the double-well potential given by Eq. (19). It is well-known that the double-well potential model which has two zeros corresponding to the vacuum states $\phi_{1}$ and $\phi_{2}$. In this case, the topological kink interpolates smoothly and monotonically between $\phi_{1}$ and $\phi_{2}$, according to Figs. I and II. But, in our non-polynomial scalar potential one builds a static, infinite-energy and classically singular field configuration, which is in a non-topological sector. Indeed, we have found a new potential model given by Eq. (20) that is solved exactly in the context of classical theory in $(1+1)$-dimensions.

In conclusion, we found $V_{1-}$ and $V_{1+}=V_{2+}$ is a supersymmetric potential pair with unbroken SUSY, so that $F_{1-}$ refers to the bosonic sector of the SUSY fluctuation operator

$F_{S U S Y}$, while $F_{1+}$ is the fermionic sector of $F_{S U S Y}$. In this case, $\eta_{1-}^{(0)}$ then becomes the unique normalizable eigenfunction of the $F_{S U S Y}$ corresponding to the zero mode of the ground state. On the other hand, the spectra to $F_{2 \pm}$ are identical and in this case, there are no zero mode for the ground state i.e. $\omega_{-}=\omega_{+}>0$, thus one has broken SUSY.

Therefore, our non-polynomial potential does not have the reflection symmetry $\phi \rightarrow$ $-\phi$, with a stability equation so that it does not lead to either a bosonic zero mode or its supersymmetric partner because both eigenfunctions are non-normalizable with SUSY broken. Thus, the scheme above for proposing new field potential models is not always physically acceptable, because it may lead to infinite energy configuration.

\section{Acknowledgments}

RLR wishes to thanks the Dr. A. N. Vaidya of the Instituto de Física of the UFRJ for the incentive and the staff of CBPF and DCEN-CFP-UFPB to facilities. This research was supported in part by CNPq (Brazilian Research Agency). RLR and VGL are grateful to Dr. J. A. Helayel-Neto for his hospitality and fruitful discussions. 


\section{REFERENCES}

[1] R. Rajaraman, Solitons and Instantons, (North-Holland, Amsterdam, 1982), p. 20-21; S. Coleman, Aspects of Symmetry, (Cambridge University, London, 1985), p. 190-191.

[2] P. G. Drazin and R. S. Johnson, Solitons: an introduction, (Cambridge University Press, Cambridge, 1993.)

[3] A. P. Balachandran, G. Marmo, B. S. Skagerstam and A. Stern, Classical Topology and Quantum States, (World Scientific, Singapore, 1991), p. 104-106.

[4] E. J. Weinberg, Annu. Rev. Nucl. Part. Sci. 42, 177 (1992).

[5] E. Witten, Nucl. Phys. B185, 513 (1981); C. V. Sukumar J. Phys. A: Math. Gen. 18, 2917 (1985);D. L. Pursey, Phys. Rev. D33, 1047 (1986); D. L. Pursey, Phys. Rev. D33, $2267(1986)$.

[6] L. É. Gendenshtein, JETP Lett. 38, 356 (1983); L. É. Gendenshtein and I. V. Krive, Sov. Phys. Usp. 28, 645 (1985); R. Dutt, A. Khare and U. P. Sukhatme, Am. J. Phys. 56, 163 (1988); A. Lahiri, P. K. Roy and B. Bagchi, Int. J. Mod. Phys. A5, 1383, (1990).

[7] M. N. Nieto, Phys. Lett. B145 208 (1984); W.-Y. Keung, U. P. Sukhatme, Q. Wang and T. D. Imbo, J. Phys. A: Math. Gen. 22, L987 (1989). Am. J. Phys. 56, 163 (1988).

[8] F. Cooper, A. Khare and U. Sukhatme, Phys. Rep. 251, 267-285, (1995).

[9] R. de Lima Rodrigues, V. B. Bezerra and A. N. Vaidya, Phys. Lett. A287, 45 (2001).

[10] R. de Lima Rodrigues, P. Barbosa da Silva Filho and A. N. Vaidya, Phys. Rev. D58, 125023 (1998).

[11] C. N. Kumar, J. Phys. A: Math. Gen. 20, 5397 (1987).

[12] R. de Lima Rodrigues Mod. Phys. Lett. A10, 1309 (1995).

[13] Q. Wang, U. P. Sukhatme and W.-Y. Keung, Mod. Phys. Lett. A5, 525 (1990); D. S. Kulshreshtha, J.-Q. Liang and H. J. W. Müller-Kirsten, Ann. Phys. (N. Y.) 225, 191 (1993).

[14] J. Casahorran and S. Nam, Int. J. Mod. Phys. A6, 5467 (1991); C. V. Sukumar, J. Phys. A: Math. Gen. 19, 2297 (1986); J. Hruby, J. Phys. A: Math. Gen. 22, 1807 (1989); L. J. Boya. and J. Casahorran, Ann. Phys. (N. Y.) 196, 361 (1989).

[15] W.-Y. Keung, U. P. Sukhatme, Q. Wang and T. D. Imbo, J. Phys. A: Math. Gen. 22, L987 (1989); G. Junker and P. Roy, Ann. of Phys. 256, 302 (1997); P. Barbosa da Silva Filho, R. de Lima Rodrigues, A. N. Vaidya, J. Phys. A: Math. Gen., 32, 2395 (1999).

[16] G. H. Flores and N. F. Svaiter, preprint Notas de Física, CBPF-NF-014/01; hepth/01017043. 


\section{FIGURES}



FIG. 1. Double-well potential $V(y)=\frac{1}{8}\left(y^{2}-4\right)^{2}$, for $\lambda=\frac{1}{4}, \quad m=1$.

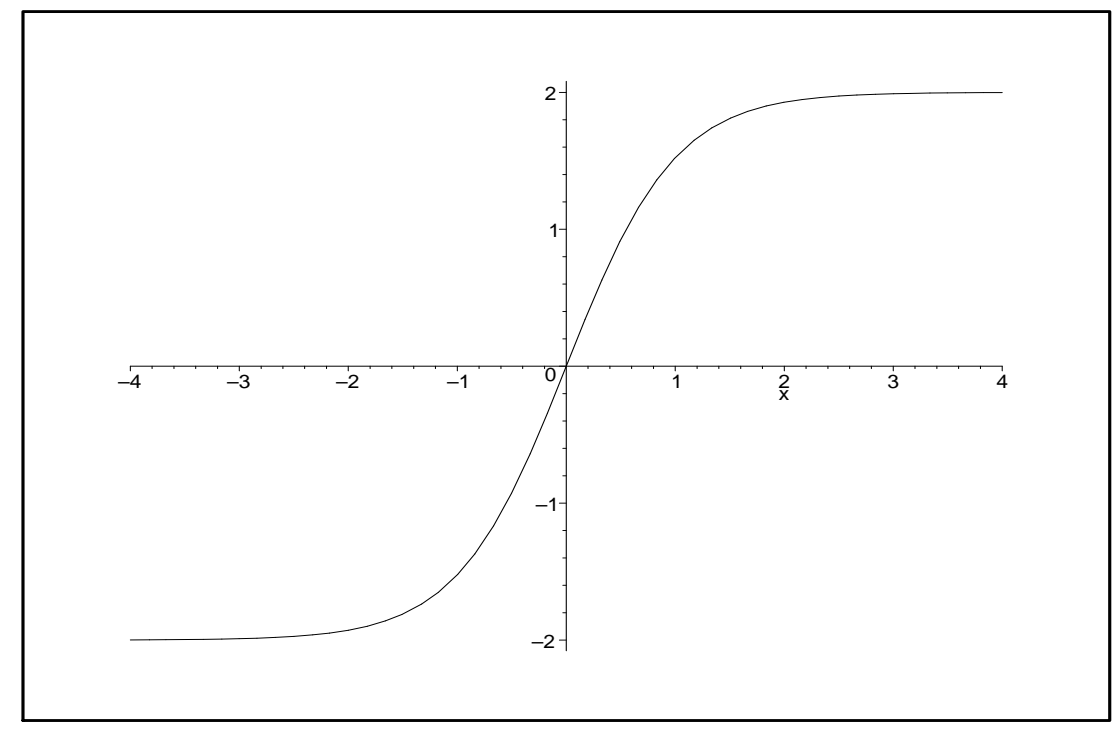

FIG. 2. The Kink $y=\phi(x)$ of the double-well potential, for $\lambda=\frac{1}{4}, \quad m=1$. 


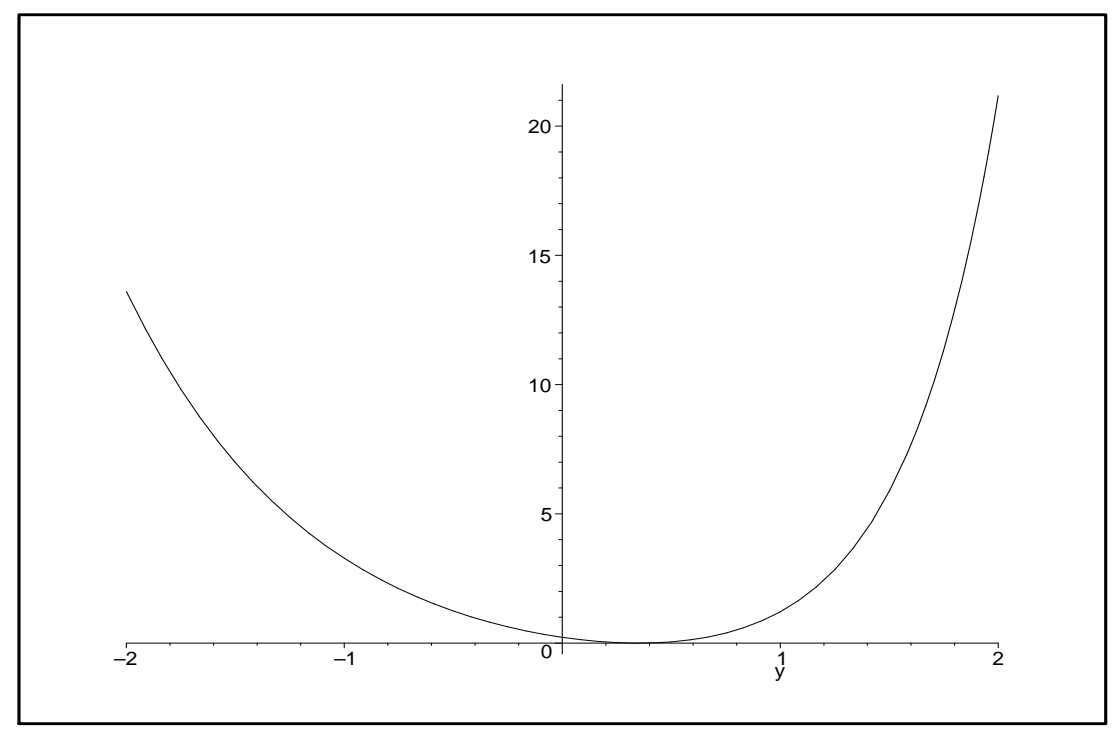

FIG. 3. Non-polynomial potential, for $\lambda=1, \quad m=1$.

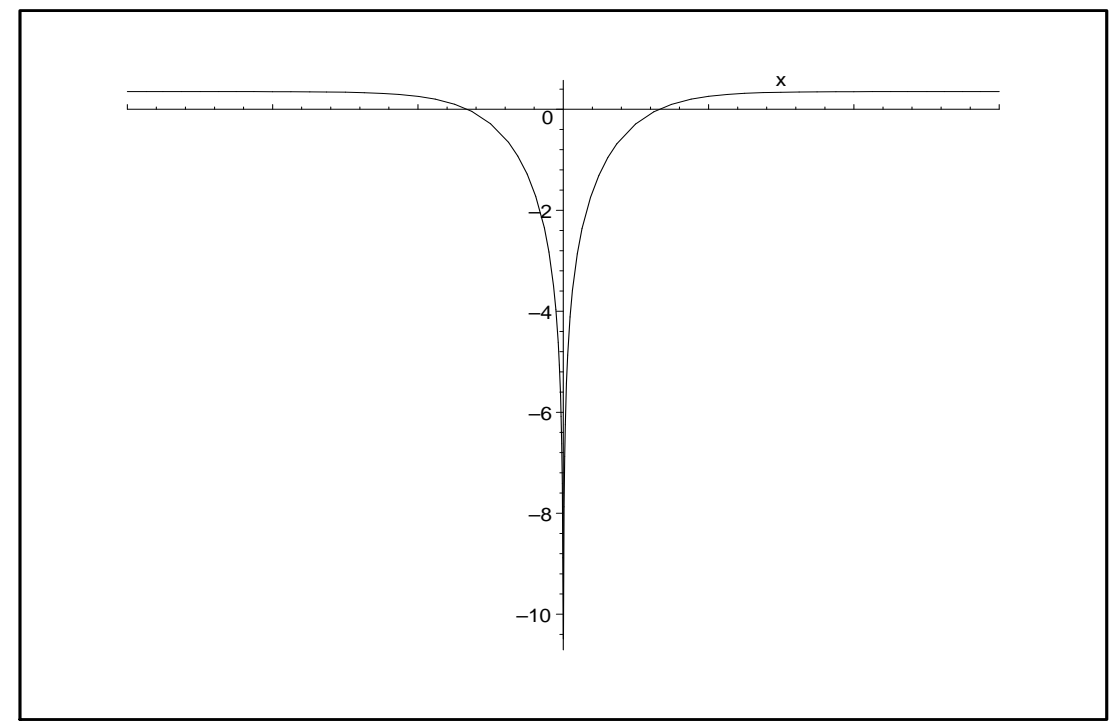

FIG. 4. Static classical configuration associated to the non-polynomial potential, for $\lambda=1, \quad m=1$. 